\title{
Odontoma - Report of Two Cases and Literature Review
}

\author{
Elitsa Deliverska, Lyudmil Gagov, Lutchezar \\ Stefanov, Martin Rubiev.
}

Department of Oral and Maxillofacial surgery, Faculty of

Dental Medicine, Medical University- Sofia;

\begin{abstract}
Background: Odontomas are mixed odontogenic tumors composed of mineralized tissue of ectomesenchymal origin. According to the World Health Organization (WHO) ranks, the odontomas are classified into two main types: complex and compound. They are usually detected in routine radiographs and may be related to various causes, and are rarely associated with impacted teeth.

Purpose: The aim is to present two clinical cases of complex odontoma that prevent appropriate eruption of permanent teeth.

Material and methods: We present two cases of complex odontoma associated with a retained permanent tooth. The orthopantomography and CBCT revealed circular and ovoid in shape amorphous radiopaque masses, surrounded by a thin radiolucent zone in association with an impacted permanent tooth.

Results: The treatment of choice was extraction of the primary first molar, enucleation of the odontoma and the preservation of the first premolar. The complete removal of the complex odontoma was successfully performed, since after few months of follow-up the maxillary permanent teeth resumed their eruptive process. The result of the biopsy confirmed cases of complex odontoma.

Conclusion: Odontomas are tumours of the dental tissues and may interfere with the eruption of the associated tooth. The early diagnosis, followed by a proper treatment at the right time, will result in a favorable prognosis and a desirable development of occlusion.
\end{abstract}

Keywords: odontoma, complex, enucleation, ectomesenchymal 


\section{Background}

According to the 2005 World Health Organization classification, an odontoma is an odontogenic benign tumor of young age (1). Despite this, odontomas are clinically considered as tumor-like formations (hamartomas of dental tissues) or developmental anomalies, rather than true odontogenic neoplasms (2). Two main types of odontoma are described: (a) complex odontoma, an amorphous and disorderly pattern of calcified dental tissues, and (b) compound odontoma, multiple miniature or rudimentary teeth (3-7). The compound odontoma has predilection toward the anterior maxilla (61\%), whereas only $34 \%$ of complex odontomas occur in this area; the complex type shows a predilection for the posterior jaws (59\%) and lastly the premolar area (7\%). Both variants are made of all dental tissues such as enamel, dentin, cementum, and pulp $(7,8)$.

Compound odontomas have numerous tooth-like structures (with altered size and shape) known as denticles. At X-ray evaluation, compound odontomas appear as well-delimited lesions with a radiotransparent halo containing radiodense zones which represent small denticles, separated by fibrous septae, while in the complex types the radiodense elements appear as irregular and disorderly masses with no similarity to dental structures $(2,9)$. These lesions are often associated with impacted permanent teeth $(10,11)$. Impaction has been defined as the prevention of the eruption of a tooth to the expected times into a normal functional position due to the presence of an obstacle or reasons of different nature (8, 12). In all cases, surgical removal represents the best therapeutic option and the prognosis after treatment is very favorable, with very low recurrence $(7,8,12,13,14,15,16,17)$.

The aim of this case report is to describe a minimally invasive surgical procedure to remove a compound odontoma localized in the maxilla area associated with unerupted permanent maxillary teeth. The purpose of this technique is to preserve as much as possible the surrounding bone tissue in order to promote healing and cause less discomfort to the patient during postoperative time.

\section{Case 1}

The treatment of choice was extraction of the primary first molar, enucleation of the odontoma and the preservation of the first premolar. The complete removal of the complex odontoma was successfully performed, since after few months of follow-up the maxillary permanent teeth resumed its eruptive process. The result of the biopsy confirmed cases of complex odontoma.

A 14-year-old male patient in an apparently good health condition was referred to the Department of Oral and Maxillofacial Surgery, Faculty of Dental Medicine, Medical University, Sofia by his orthodontist for the absence of the right upper permanent second premolar. The subject had no significant medical history and had not reported oral trauma or infections. Intraoral examination showed the presence of the primary right second molar over the physiological period of exfoliation, which meant that there was no correspondence between chronological and dental ages.

A radiographic examination (panoramic X-ray, fig.1) showed an amorphous mass of calcified material with the radiodensity of tooth structure, which bears no anatomical resemblance to a tooth, surrounded by a narrow radiolucent rim and the unerupted right second premolar in upper standing. 


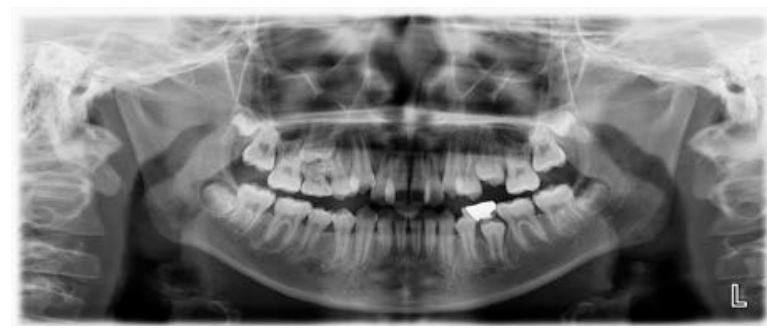

Fig.1 Ortopantomography

The provisional diagnosis of complex odontoma.

A computed tomography (CT) with the Dentascan program (Siemens Rs Somaton Volume Zoom Kv $120 \mathrm{~mA}$ 140; Siemens, Erlangen, Germany) was performed in order to define the extension of the lesion and the anatomical topography, showing the unerupted permanent tooth. cranially and mesially positioned compared to odontoma.(Fig.2)
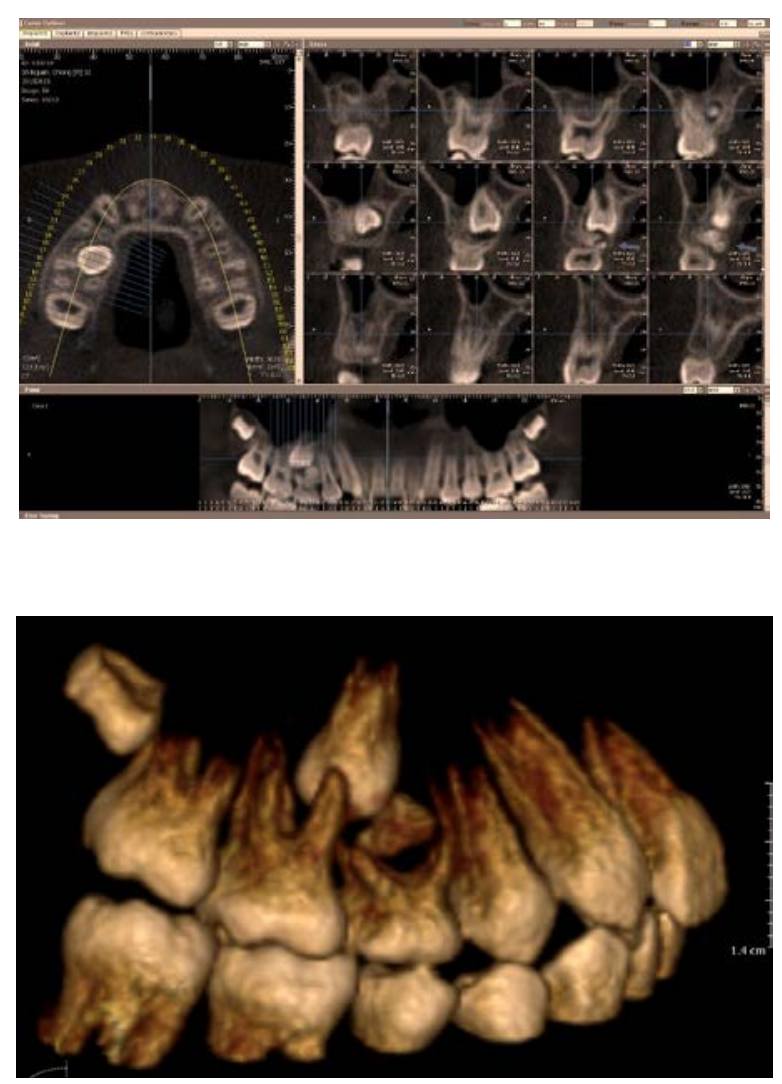

Fig. 2 CT scan of complex odontoma

In accordance with the patient and his orthodontist, surgical removal of the odontoma and exposure of the associated impacted second premolar was planned. Surgery was performed under local anesthesia (4\% articain with 1:100,000 epinephrine). The extraction of the deciduous second molar was also performed. A mucoperiosteal flap was etched and raised and bone was removed on the vestibular side using a low- 
speed dental hand-drill and a tungsten carbide bur so that the crown of the permanent impacted second premolar was exposed. The odontoma was removed. The wound was carefully irrigated with physiological solution and cleaned with a sterile dressing; the flap was repositioned and sutured with 3.0 absorbable suture (Vicryl, Johnson \& Johnson, Sint-Stevens-Woluwe, Belgium).

The histological examination confirmed the clinical and radiographic diagnosis of complex odontoma (Specimen No 32619/02.2016).

The postoperative period was uneventful. Postoperative treatment consisted of amoxicillin and clavulanic acid $(0,625 \mathrm{~g}$ twice a day for 5 days), paracetamol ( $500 \mathrm{mg}$ twice a day for 2 days, and then as needed), and Tantum Verde spray.

The patient was referred to the orthodontist to continue the treatment.

A 12-year-old boy reported seeking treatment for his unerupted maxillary permanent left second molar. His medical and family history was non-contributory. Intraoral examination revealed the clinical absence of a maxillary left second molar, while the contralateral tooth was in an eruption process and normally positioned in the arch. The radiographic examination (Fig. 3) revealed the presence of an impacted maxillary left second molar. There was a well-defined radiodense mass adjacent to and partially overlapping the coronal portion of the unerupted second molar. It was surrounded by a thin radiolucent zone measuring approximately $0.5 \times 0,5 \mathrm{~cm}$.

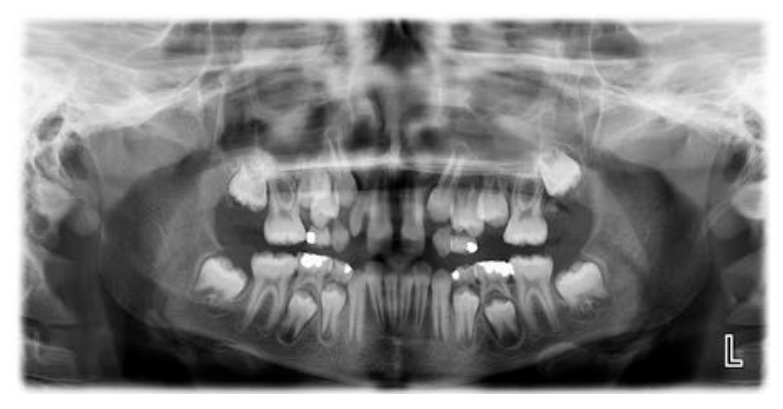

Fig.3 Ortopantomography of odontoma in left maxilla.

In accordance with the clinical and radiographic examination, a provisional diagnosis of odontoma was made. The treatment consisted of conservative surgical removal of the lesion and clinical and radiographic follow-up. After achieving local anesthesia, a mucoperiosteal triangular flap was reflected from the maxillary first left molar to the distal aspect of it. A layer of bone overlying the tumor and the unerupted molar was removed using a round surgical bur under constant irrigation with saline as a coolant. The odontoma was removed along with the fibrous capsule, without disturbing the unerupted permanent molar. The surgical site was curetted and irrigated with a povidone iodine--saline solution. After hemostasis was achieved, the flap was approximated and closed primarily with 3--0 silk sutures. The specimen was placed in $10 \%$ formalin and sent for histopathological examination, which confirmed the provisional diagnosis of complex odontoma. One week later the sutures were removed, with normal healing being observed. After complete healing, a functional removable space maintainer was constructed and delivered. The patient was advised for routine clinical and radiographic follow-up once in 3 months, to assess the eruption of the unerupted tooth and to examine for the recurrence of odontoma. 


\section{Discussion}

The term "odontoma" was introduced by Paul Broca in 1867 to describe "tumors formed by the overgrowth of transitory or complete dental tissues." Odontomas are intraosseous lesions mainly located in the anterior maxilla and anterior mandible, although lesions localized in gingival soft tissues have also been reported $(7,16)$. The majority of odontomas are asymptomatic, although swelling, pain, suppuration, bony expansion, and displacement of teeth have been rarely observed. They frequently lead to impaction or delayed eruption of permanent teeth. $(11,12)$ Their pathogenesis has been associated with a number of causes including trauma during primary dentition $(9,17)$, hereditary anomalies such as Gardner's syndrome, Hermann's syndrome, and basal cell nervous syndrome, odontoblastic hyperactivity, or alterations of the genetic components responsible for controlling dental development $(8,16)$. The development of the odontoma is commonly associated with eruption failure of permanent teeth, impaction, and delayed exfoliation of primary teeth $(17,18,19,20,21)$. In these cases, the presence of odontoma prevented the physiological eruption of permanent maxillary teeth. In accordance with the literature, the patient had no pain but the lesion's pathogenesis remained unknown: there were no reported previous traumatic or infective episodes and the medical history was negative.

The treatment of choice for compound odontomas is surgical removal, followed by histopathological analysis to confirm the diagnosis $(7,22,23,24,25)$. According to the literature, the optimal management of the impacted tooth should allow its conservation and repositioning in the arch $(22,23,24,25,26,27)$. On the other hand, impacted teeth are frequently reported to be extracted simultaneously with the odontoma $(25,28,29)$. In this case, the permanent teeth were in good position and we saved them and expect future eruption.

In these cases, the removal of odontoma was followed by the extraction of the deciduous tooth. The surgical difficulty was determined by the necessity to adopt a technique as conservative as possible in prediction of the subsequent eruption of permanent tooth. Therefore, the bone tissue removal around the wound was minimized, making the surgical steps of lesion and tooth removal more complicated.

A careful evaluation with panoramic and CT Dentascan X-rays revealed a buccal and cranial positioning of the permanent premolar to the odontoma. For this reason, a vestibular access was chosen, creating a small bone fenestration with the use of low-speed dental hand-drill and a tungsten carbide bur to extract the odontoma. This conservative approach allowed saving the precious bone ridge and avoiding the formation of tissue defects.

A histological analysis was finally taken in order to confirm the odontoma's diagnosis.

In conclusion, the presence of odontoma in association with the impacted canine needs an early diagnosis and a surgical removal treatment. Profound knowledge and an excellent evaluation of X-ray documents are essential for adequately resolving each clinical case. The adoption of a conservative surgical approach is advisable, in order to preserve the dental tissues and obtain optimal tissue healing. A histological evaluation is necessary to confirm the correct diagnosis of odontoma.

Clinical experience suggests and the dental literature supports that suggestion that an individualized radiographic examination of any pediatric patient that presents clinical evidence of delayed permanent tooth eruption or temporary tooth displacement with or without a history of previous dental trauma should 
be performed. As was demonstrated by this report, early diagnosis of odontomas allows the adoption of a less complex and less expensive treatment and ensures better prognosis.

\section{References}

1. Hidalgo-Sánchez O, Leco-Berrocal MI, Martínez-González JM. Metaanalysis of the epidemiology and clinical manifestations of odontomas. Med Oral Patol Oral Cir Bucal. 2008;13:E730-734.

2. Rajendran R, Sivapathasundharam B. Shafer's text book of oral pathology. 5th ed. Amsterdam: Elsever; 2006. Cysts and tumors of odontogenic origin.Disturbances of development and growth. Shafer, Hine, Levy; pp. 357-432.

3. Batra P, Duggal R, Kharbanda OP, Parkash H. Orthodontic treatment of impacted anterior teeth due to odontomas: A report of two cases. J Clin Pediatr Dent. 2004;28:289-294.

4. Prætorius F, Piattelli A. Odontoma, Odontogenic tumors. In: Barnes L, Eveson JW, Reichart P, Sidransky D, editors. WHO Classification of tumors. Pathology and Genetics of Head and Neck Tumors. Lyon France: IARC Press; 2005. pp. 310-311.

5. Yeung $\mathrm{KH}$, Cheung RC, Tsang MM. Compound odontoma associated with an unerupted and dilacerated maxillary primary central incisor in a young patient. Int J Paediatr Dent. 2003;13:208212.

6. Boffano P, Zavattero E, Roccia F, Gallesio C. Complex and compound odontomas. Journal of Craniofacial Surgery. 2012, vol. 23, no. 3, pp. 685-688.

7. Yassin OM. Delayed eruption of maxillary primary cuspid associated with compound odontoma. J Clin Pediatr Dent. 1999;23:147-149.

8. Amailuk P, Grubor D. Erupted compound odontoma: case report of a 15-year-old Sudanese boy with a history of traditional dental mutilation. Br Dent J. 2008; 204:11-14.

9. Tomizawa M, Otsuka Y, Noda T. Clinical observations of odontomas in Japanese children: 39 cases including one recurrent case. Int J Paediatr Dent. 2005;15:37-43.

10. Hattab FN, Yassin OM, Rawashdeh MA. Supernumerary teeth: Report of three cases and review of the literature. J Dent Child. 1994;61:382-393.

11. Neville BW, Damm DD, Allen CM, Bouquot JE: Oral and Maxillofacial Pathology. Philadelphia: Saunders, 1995, pp 531-533.

12. Cawson RA, Binnie WH, Eveson JW: Color Atlas of Oral Disease. Clinical and Pathological Correlations. Hong Kong: Mosby-Wolfe, 1993, pp. 6-19.

13. Owens BM, Schuman NJ, Mincer HH, Turner JE, Oliver FM: Dental odontomas: a retrospective study of 104 cases. J Clin Pediatr Dent 21:261-264, 1997. 
14. Katz RW: An analysis of compound and complex odonto- mas. ASDC J Dent Child 56:445449, 1989.

15. Bengston AL, Bengston NG, Benassi, LRDC: Odontomas em pacientes pediátricos. Revista de Odontopediatria 2:25-33, 1993.

16. Budnick SD: Compound and complex odontomas. Oral Surg Oral Med Oral Path 42:501-506, 1976.

17. Shafer WG, Hine MK, Levy BM: A Textbook of Oral Pathology, 4th Ed. Philadelphia:Sanders, 1983,pp. 308-311.

18. Areal-López L, Silvestre DF, Gil LJ: Compound odontoma erupting in the mouth: 4 year followup of a clinical case. J Oral Pathol 21:285-288, 1992.

19. Shulman E, Corio RL: Delayed eruption associated with an odontoma. ASDC J Dent Child 54:205-207, 1987.

20. Brunetto AR, Turley PK, Brunetto AP, Regattieri LR, Nicolau GV: Impaction of a primary maxillary canine by an odontoma: surgical and orthodontic management. Pediatr Dent 13:301302, 1991.

21. Gallien GS, Schuman NJ, Sharp HK, Mcllveen LP: Odontoma of a maxillary central incisor in a 10-year-old black male. J Pedod 10:352-355, 1986.

22. Oliver RG, Hodges CGL: Delayed eruption of a maxillary central incisor associated with an odontome: report of case. ASDC J Dent Child 55:368-371, 1988. 23. Thwaites MS, Camacho JL: Complex odontoma: report of case. ASDC J Dent Child 54:286-288, 1987.

24. Giunta JL, Kaplan MA: Peripheral, soft tissues odontomas. Oral Surg Oral Med Oral Pathol 69:406-411, 1990.

25. Philipsen HP, Reichart PA, Praetorius F: Mixed odontogenic tumours and odontomas. Considerations on interrelashionship. Review of the literature and presentation of 134 new cases of odontomas. Oral Oncology 33:86-99, 1997.

26. Takeda Y: Ameloblastic fibroma and related lesions:current pathology concept. Oral Oncology 35:535-540, 1999.

27. Wright,G, Starkey, PE, Gadner, DE: Child Management in Dentistry. Bristol: Wright, 1987, pp. 81-82.

28. Kajiyama $\mathrm{K}$, Kai $\mathrm{H}$ : Esthetic management of an unerupted maxillary central incisor with a closed eruption technique. Am J Orthod Dentofacial Orthop 2000,118:224-228.

29. Kaban, LB: Pediatric Oral and Maxillofacial Surgery. Philadelphia: Saunders, 1990, pp. 111112. 


\section{Corresponding author:}

Associate prof. Elitsa Deliverska, PhD

Sofia 1431 Str."Georgi Sofiiski"1

Faculty of Dental medicine

Medical University Sofia

Department of Oral and Maxillofacial surgery 